\title{
Microsatellite markers uncover cryptic species of Odontotermes (Termitoidae: Termitidae) from Peninsular Malaysia
}

\begin{abstract}
Termites from the genus Odontotermes are known to contain numerous species complexes that are difficult to tell apart morphologically or with mitochondrial DNA sequences. We developed markers for one such cryptic species complex, that is, Odontotermes srinakarinensis sp. nov. from Maxwell Hill Forest Reserve (Perak, Malaysia), and characterised them using a sample of 41 termite workers from three voucher samples from the same area. We then genotyped 150 termite individuals from 23 voucher samples/colonies of this species complex from several sites in Peninsular Malaysia. We analysed their population by constructing dendograms from the proportion of shared-alleles between individuals and genetic distances between colonies; additionally, we examined the Bayesian clustering pattern of their genotype data. All methods of analysis indicated that there were two distinct clusters within our data set. After the morphologies of specimens from each cluster were reexamined, we were able to separate the two species morphologically and found that a single diagnostic character found on the mandibles of its soldiers could be used to separate the two species quite accurately. The additional species in the clade was identified as Odontotermes denticulatus after it was matched to type specimens at the NHM London and Cambridge Museum of Zoology.
\end{abstract}

Keyword: Odontotermes; Microsatellites; Macrotermitinae; Cryptic species; Taxonomy 